\title{
Conjugalidade e representações sociais do HIV/AIDS pela equipe multiprofissional de saúde
}

\author{
Conjugality and a multi-professional health team's social representations of HIV/AIDS \\ Conyugalidad y representaciones sociales del SIDA/VIH por el equipo de salud \\ multiprofesional
}

\author{
Mariana de Sousa Dantas'; Rebeca Coelho de Moura Angelim"I; \\ Fátima Maria da Silva Abrão ${ }^{\text {III }}$; Denize Cristina de Oliveira ${ }^{I V}$
}

\begin{abstract}
RESUMO: Objetivou-se analisar os conteúdos e as estruturas das representações sociais do Vírus da Imunodeficiência Humana/ Síndrome da Imunodeficiência Adquirida (HIV/AIDS) pela equipe multiprofissional de saúde, apresentada pelos profissionais em geral, incluindo, neste escopo, aqueles com companheiro(a). Estudo descritivo, de abordagem qualitativa e estrutural da Teoria das Representações Sociais. Realizado entre 2011 e 2013, com 86 profissionais de saúde em Recife, Brasil. Mediante questionário, a análise de evocações livres apresentou os quadros de quatro casas dos profissionais. Como resultados, preconceito assume dimensão afetiva-atitudinal negativa; tratamento associado à adesão-tratamento e prevenção denotam o saber clínico acerca do fenômeno. No grupo com companheiro(a), doença apareceu no $4^{\circ}$ quadrante e prevenção no $3^{\circ}$ mantendo o caráter clínico incorporado à síndrome. As representações caracterizam a abordagem clínica/medicamentosa e o estigma, traduzido pelo preconceito social.
\end{abstract}

Palavras-Chave: Representações sociais; síndrome da imunodeficiência adquirida; pessoal de saúde; comportamento sexual.

\begin{abstract}
Taking a descriptive, qualitative, structural approach to social representations theory, this study examined the content and structures of a multidisciplinary health team's social representations of HIV/AIDS, as expressed by the team in general and by those members with a partner. Analysis of free evocations in response to a questionnaire applied to 86 health professionals in Recife, Brazil, between 2011 and 2013, resulted in a corresponding four-quadrant chart in which prejudice displayed a negative affective and attitudinal quality; while treatment, associated with treatment adherence and prevention, denote clinical knowledge about the phenomenon. Among those with a partner, disease appeared in the $4^{\text {th }}$ quadrant and prevention in the $3^{\text {rd }}$, maintaining the clinical view of the syndrome. The representations characterize the clinical/medicine approach and stigma, expressed as social prejudice.

Keywords: Social representations; acquired immunodeficiency syndrome; health personnel; sexual behavior

RESUMEN: Este estudio tuvo como objetivo el análisis del contenido y de las estructuras de las representaciones sociales del Virus de la Inmunodeficiencia Humana/Síndrome de la Inmunodeficiencia Adquirida (VIH/SIDA) por el equipo multidisciplinario de salud, presentado por profesionales en general y aquéllos que tienen pareja. La investigación tuvo un enfoque descriptivo, cualitativo y estructural de la Teoría de las Representaciones Sociales. Se llevó a cabo entre los años 2011 y 2013, junto a 86 profesionales de salud en Recife, Brasil. Por medio de cuestionario, el análisis de las evocaciones libres presentó los cuadros de cuatro casas de los profesionales. Como resultados, prejuicio asume dimensión afectiva/actitudinal negativa; tratamiento asociado con adhesión-tratamiento y prevención denotan el conocimiento clínico sobre el fenómeno. Dentro del grupo con pareja, enfermedad apareció en el cuarto cuadrante y prevención en el tercero, manteniendo el carácter clínico incorporado en el síndrome. Las representaciones caracterizan el enfoque clínico/farmacológico y el estigma, traducido por el prejuicio social. Palabras Clave: Representaciones sociales; síndrome de inmunodeficiencia adquirida; personal de salud; comportamiento sexual.
\end{abstract}

\section{INTRODUÇÃO}

A infecção pelo Vírus da Imunodeficiência Humana (HIV) tem se manifestado de forma global no decorrer dos 30 anos da sua descoberta. Para tanto, configura-se uma epidemia heterogênea, cuja progres- são ocorre, geográfica e socialmente, em locais e grupos populacionais específicos, como usuários de drogas injetáveis, homens que fazem sexo com homens e profissionais do sexo nos diversos continentes do planeta ${ }^{1}$.

'Enfermeira. Mestre em Enfermagem pelo Programa Associado de Pós-Graduação em Enfermagem da Universidade de Pernambuco e Universidade Estadual da Paraíba. Recife, Pernambuco, Brasil. E-mail: nanasdantas@ @hotmail.com.

IEnfermeira. Mestranda em Enfermagem pelo Programa Associado de Pós-Graduação em Enfermagem da Universidade de Pernambuco e Universidade Estadual da Paraíba. Recife, Pernambuco, Brasil. E-mail: rebecaangelim@hotmail.com.

IIIEnfermeira. Doutora em Enfermagem. Coordenadora e Docente do Programa Associado de Pós-Graduação em Enfermagem da Universidade de Pernambuco e Universidade Estadual da Paraíba. Recife, Pernambuco, Brasil. E-mail: abraofatima@gmail.com.

IVEnfermeira. Doutora em Saúde Pública. Professora Titular da Universidade do Estado do Rio de Janeiro. Brasil. E-mail: dcouerj@gmail.com. 
No que concerne à evolução da epidemia da Síndrome de Imunodeficiência Adquirida (AIDS) no contexto global, dados do Programa Conjunto das Nações Unidas sobre HIV/AIDS (UNAIDS) apontaram que, em 2012, existiam 35,3 milhões de pessoas com HIV, sendo 2,3 milhões de pessoas com novas infecções e 1,6 milhão de mortes por complicações ligadas à doença ${ }^{2}$.

Segundo relatório oficial do referido Programa, acredita-se que o acesso à terapia antirretroviral tem contribuído para o declínio no número de novas infecções desde 2001. Contudo, o fenômeno apresenta desafios significativos, tendo em vista a alta morbimortalidade e discriminação enfrentada pelas pessoas que vivem com o HIV/AIDS (PVHA) ${ }^{2}$. Desse modo, ressalta-se a importância da participação da sociedade civil na resposta à epidemia de AIDS em todos os países.

No Brasil, os dados evidenciaram 39.185 casos da doença em 2012. Entre as capitais da Região Nordeste, a cidade do Recife ocupa a segunda posição relacionada às maiores taxas de incidência, o que corresponde a 39 casos para cada 100.000 habitantes $^{3}$.

Convém destacar que o progresso do fenômeno foi determinante para que o HIV/AIDS fosse considerado objeto social, desde a evolução do não familiar para algo familiar, propiciando o surgimento de diversas representações ${ }^{4}$.

Para tanto, é oportuno realizar um estudo acerca das representações sociais do HIV/AIDS por profissionais da equipe de saúde, tendo em vista a escassez de pesquisas científicas envolvendo esse grupo. Além disso, merece destaque a complexidade da abordagem sobre o vínculo conjugal, cujas condutas pessoais e inter-relações podem sofrer influências sociais, econômicas e culturais.

Em relação à conjugalidade, convém destacar a importância do desenvolvimento de estudos sobre essa temática, focalizando a dinâmica interacional, capaz de fomentar teorias e práticas clínicas mais contextualizadas 5 .

As relações conjugais, ao serem compreendidas no sentido da conjugação de individualidades entre o casal, incitam a necessidade de uma melhor compreensão das diversas abordagens, incluindo os norteamentos psicológicos abrangidos por estudos dessa natureza ${ }^{6}$.

Ante ao exposto, cabe destacar estudo realizado com enfermeiros, cujas representações sociais sobre a vulnerabilidade permeiam as relações interpessoais, denotando o desuso do preservativo e a invisibilidade do HIV nas relações afetivo-sexuais?

Desse modo, essas proposições sugerem o seguinte questionamento: Quais os conteúdos representacionais do HIV/AIDS atribuídos por profissionais da equipe de saúde, no contexto da conjugalidade? O presente estudo tem como objetivo analisar os conteúdos e as estruturas das representações sociais do HIV/AIDS pela equipe multiprofissional de saúde, apresentada pelos profissionais em geral e aqueles com companheiro(a).

\section{Referencial Teórico-Metodológico}

Este estudo faz parte do Projeto Multicêntrico, intitulado As transformações do cuidado de saúde e enfermagem em tempos de AIDS: representações sociais e memórias de enfermeiros e profissionais de saúde no Brasil ${ }^{8}$.

As reflexões acerca do HIV/AIDS manifestam-se pelo destaque que o processo saúde-doença assume no campo de investigação social, tornando oportuna a utilização da Teoria das Representações Sociais (TRS) nos diversos âmbitos de pesquisa, pois ela propicia a produção de alternativas teóricas que contemplam o diferente, o não habitual, o inesperado a partir de um contexto intercambiável de respostas e modos de pensar?.

Corroborando com essa perspectiva, a construção do conhecimento acerca do processo saúde-doença tem sido influenciada pelas ciências humanas e sociais. Assim, o uso da TRS permite a formulação da representação social de um determinado objeto e possibilita a compreensão, organização e adequação da prática profissional e assistencial ${ }^{10}$. Nesse prisma, a construção das representações é influenciada pela relação entre os grupos sociais, estabelecida entre a pessoa soropositiva, profissionais de saúde e sociedade em geral ${ }^{11}$.

Trata-se de um estudo descritivo, com abordagem qualitativa, baseada na vertente estrutural, elaborada por Jean-Claude Abric, da Teoria das Representações Sociais. Realizado no período de 2011 a 2013, em rede de serviços de saúde em HIV/AIDS na cidade de Recife, Pernambuco, Brasil. A amostra constituiu-se de 86 trabalhadores selecionados de forma intencional, incorporados à equipe multiprofissional, cujas práticas assistenciais direcionadas às pessoas acometidas pelo HIV/AIDS ocorreram no período superior a seis meses, em Serviços de Assistência Especializada (SAE) e Centro de Testagem e Aconselhamento (CTA).

Aplicou-se um questionário composto por variáveis de ordem sociodemográfica, bem como pelas evocações livres. Os dados analisados com o auxílio da Técnica de Evocações Livres asseguraram a elaboração do quadro de quatro casas, através da versão 2005 do software Ensemble de Programmes Permettantl Analyse des Evocations (EVOC), buscando identificar a estruturação dos conteúdos representacionais ${ }^{12}$.

Sabe-se que essa técnica, também denominada análise prototípica, permite que as projeções mentais dos participantes sejam apreendidas de maneira rápida, objetiva e espontânea, or meio da citação de três a cinco palavras ou expressões que possam imaginar acerca de um ou mais termos indutores (estímulos) detectados pela audição ou visualização, para assimilar a noção da realidade $^{12-14}$. Nesse contexto, para o presente estudo, utilizou-se o termo indutor HIV/AIDS, solicitando-se cinco palavras ou expressões que viessem à mente dos 
profissionais de saúde ao pensar nesse termo indutor.

Ressalta-se que o estudo obedeceu aos requisitos éticos e legais contidos na Resolução no 466/2012, do Conselho Nacional de Saúde ${ }^{15}$. $\mathrm{O}$ projeto de pesquisa foi aprovado pelo Comitê de Ética e Pesquisa da Universidade de Pernambuco (UPE), sob Certificado de Apresentação para Apreciação Ética (CAAE) de número 01080.0.097.000-11, sendo mantidos o caráter sigiloso e a integridade pessoal dos profissionais de saúde que assinaram o Termo de Consentimento Livre e Esclarecido.

\section{Resultados e Discussão}

No presente estudo, os dados sociodemográficos dos integrantes da equipe multiprofissional de saúde foram expressos pelas variáveis: função desempenhada na equipe, sexo, idade, setor de atuação e tempo de atuação destinado às PVHA, conforme explícito na Tabela 1 .

Ante ao exposto, percebe-se que a equipe multiprofissional estudada foi constituída por $74(86 \%)$ trabalhadores do SAE, sendo 69(80,2\%) representantes

TABELA 1: Distribuição dos participantes quanto à função, ao sexo, à idade, ao setor e tempo de atuação. Recife, Brasil, 2013.

\begin{tabular}{lcc}
\hline Variáveis & $\mathbf{f}$ & $\%$ \\
\hline Função & & \\
Médico (a) & 24 & 27,9 \\
Técnico de Enfermagem (a) & 14 & 16,3 \\
Psicólogo (a) & 14 & 16,3 \\
Assistente social & 10 & 11,6 \\
Enfermeiro (a) & 8 & 9,3 \\
Cirurgião dentista (a) & 4 & 4,7 \\
Farmacêutico (a) & 3 & 3,5 \\
Nutricionista & 3 & 3,5 \\
Biomédico (a) & 3 & 3,5 \\
Outros & 3 & 3,5 \\
Sexo & & \\
Feminino & 69 & 80,2 \\
Masculino & 17 & 19,8 \\
Idade & & \\
Menos de 35 anos & 20 & 23,3 \\
36 a 45 anos & 21 & 24,4 \\
46 a 55 anos & 34 & 39,5 \\
Mais de 56 anos & 11 & 12,8 \\
Setor & & \\
SAE & 74 & 86,0 \\
CTA & 12 & 14,0 \\
Tempo de atuação & & \\
Menos de 5 anos & 35 & 40,7 \\
16 a 25 anos & 29 & 33,7 \\
Mais de 26 anos & 19 & 22,1 \\
Total & 86 & 100 \\
\hline
\end{tabular}

femininas; 34(39,5\%) membros possuíam idade variando entre 46 e 55 anos e 64(74,4\%) com tempo de atuação junto às PVHA no período de até 15 anos de serviço. Em relação à categoria profissional, destacam-se 24(27,9\%) médicos, seguindo-se 14(16,3\%) técnicos de enfermagem e 14(16,3\%) psicólogos como a maior parcela do grupo estudado.

Quanto à aplicação do EVOC, os resultados são mostrados em quadros de quatro casas, expressando a estrutura representacional do HIV/AIDS para o conjunto de 86 membros da equipe multiprofissional e para 54 trabalhadores vivendo com companheiro(a), nos tópicos a seguir.

Significados do HIV/AIDS para a equipe multiprofissional de saúde

Os significados do HIV/AIDS para a equipe multiprofissional de saúde foram apresentados na Figura 1, através dos resultados da análise de evocações do grupo de 86 participantes da equipe de saúde que presta assistência às PVHA. A aplicação do teste reuniu 439 palavras associadas ao termo HIV/AIDS, sendo 145 palavras diferentes. Desse modo, foi determinada a frequência mínima (6), a frequência média das palavras evocadas (12), assim como o Rang Médio $(3,0)$, que corresponde à média de posição das palavras utilizadas para análise.

A identificação dimensional das evocações nos quadrantes expõe os termos preconceito, esperança, superação, tristeza, medo, luta, abandono, sofrimento como elementos atitudinais e afetivos; tratamento, adesãotratamento, prevenção, ajuda, cuidado e doença crônica caracterizam uma abordagem conceitual e de conhecimento sobre o objeto. Já as evocações doença e morte determinam uma dimensão imagética acerca do HIV/ AIDS, como pode ser observado na Figura 1.

Em relação à organização e à estrutura das representações sociais, essas apresentam elementos periféricos expostos nos quadrantes superior e inferior direito, os quais são resistentes às transformações, que circundam e protegem o núcleo central, além de conferir significados sobre uma determinada representação ${ }^{16}$. Por sua vez, a zona de contraste engloba palavras com baixas frequências; entretanto, foram lembradas primeiro e podem despertar o interesse do pesquisador ${ }^{13}$.

Significados do HIV/AIDS para os profissionais que vivem com companheiro (a)

Os resultados da análise de evocações referemse ao grupo composto por 54 trabalhadores da equipe multiprofissional que vivem com companheiro(a), conforme apresentados na Figura 2, que ilustra os componentes representacionais dispostos em cada quadrante.

Nesse contexto, os profissionais desse grupo evocaram um total de 269 palavras relacionadas ao estímulo indutor HIV/AIDS e 102 palavras diferentes. 
Utilizou-se frequência mínima (6), frequência média das palavras evocadas (12) e o Rang Médio $(3,0)$.

A análise dimensional das evocações mantém os elementos atitudinais e afetivos preconceito, medo; a abordagem conceitual e de conhecimento acerca do HIV/AIDS expressa por tratamento, adesão-tratamento, prevenção, cuidado e as evocações morte, doença numa dimensão imagética sobre o fenômeno. Ver Figura 2.

Os resultados apontaram um discreto deslocamento de alguns elementos representacionais do HIV/ AIDS pelos profissionais com companheiro(a) entre os quadrantes, quando comparados ao quadro de quatro casas composto pelas evocações do conjunto da equipe multiprofissional. Assim, a expressão doença foi deslocada do primeiro quadrante para a zona de contraste, parecendo constituir um subgrupo do possível núcleo central tratamento e o elemento prevenção apresentou uma mobilidade periférica do segundo para o terceiro quadrante. Apesar disso, manteve-se o caráter clínico e de estigmatização que os integrantes desse grupo incorporaram acerca da doença.

Entre os elementos periféricos, observa-se uma redução de termos para o grupo com companheiro(a). Outrossim, permaneceram as palavras adesão-tratamento na primeira periferia e morte na segunda periferia, assumida pelo aspecto incurável da enfermidade.

A organização de uma representação social apresenta uma modalidade particular, específica, a qual tanto os elementos da representação são hierarquizados como também toda representação está organizada ao redor do núcleo central, constituído por vários elementos que dão significado à representação ${ }^{16}$. Desse modo, o possível núcleo central da representação do HIV/ AIDS foi apresentado no quadrante superior esquerdo pelos elementos de maior frequência e evocação mais imediata preconceito e tratamento.

núcleo central sinaliza os elementos mais frequentes e importantes, podendo agrupar expressões menos significativas, sinônimos e protótipos associados ao objeto. Esses elementos aparecem, ainda, através da percepção social, constituída do pensamento social, que permitem pôr em ordem e entender a realidade vivida pelos indivíduos ${ }^{16}$.

Destaca-se que, entre os termos centrais, preconceito foi o mais frequentemente evocado pelos participantes, numa dimensão atitudinal negativa, associada ao conteúdo imagético apresentado pelo léxico de contraste doença. Refere-se ao preconceito social que diversos grupos estabeleceram acerca do HIV/AIDS desde o seu aparecimento. Esse termo expressa, supostamente, atitudes discriminatórias da sociedade perante as PVHA. Desse modo, identifica-se a permanência de uma atitude negativa frente à doença, podendo provocar um processo de estigmatização das PVHA.

Nesse prisma, as representações sobre a doença fundamentam-se nos significados e conhecimentos

\begin{tabular}{|clcclll|}
\hline \multirow{2}{*}{$\begin{array}{c}\text { O.M.E. } \\
\text { Freq. Med. }\end{array}$} & Termo evocado & Freq. & O.M.E. & Termo evocado & Freq. & O.M.E. \\
\hline \multirow{2}{*}{$\geq 12$} & preconceito & 47 & 2,128 & adesão- & 20 & 3,200 \\
& tratamento & 22 & 2,409 & tratamento & 16 & 3,188 \\
& doença & 16 & 2,063 & prevenção & & \\
& medo & 11 & 2,727 & & & \\
& cuidado & 10 & 2,700 & esperança & 8 & 3,500 \\
& luta & 7 & 2,857 & morte & 8 & 3,500 \\
$<12$ & sofrimento & 7 & 2,147 & superação & 7 & 3,286 \\
& abandono & 6 & 2,833 & ajuda & 7 & 3,000 \\
& medicamento & 6 & 2,667 & tristeza & 6 & 3,000 \\
& doença-crônica & 6 & 1,500 & & & \\
\hline
\end{tabular}

FIGURA 1: Análise do termo indutor "HIV/AIDS" das evocações de profissionais da equipe multiprofissional de saúde. Recife, PE, Brasil, 2013.

\begin{tabular}{|c|c|c|c|c|c|c|}
\hline \multirow{2}{*}{$\begin{array}{l}\text { O.M.E. } \\
\text { Freq. Med. }\end{array}$} & \multicolumn{2}{|c|}{$<3,0$} & \multirow[b]{2}{*}{ O.M.E. } & \multicolumn{3}{|c|}{$\geq 3,0$} \\
\hline & $\begin{array}{c}\text { Termo } \\
\text { evocado }\end{array}$ & Freq. & & $\begin{array}{c}\text { Termo } \\
\text { evocado }\end{array}$ & Freq. & O.M.E. \\
\hline$\geq 12$ & $\begin{array}{l}\text { preconceito } \\
\text { tratamento }\end{array}$ & $\begin{array}{l}28 \\
14\end{array}$ & $\begin{array}{l}1,964 \\
2,286\end{array}$ & $\begin{array}{l}\text { adesão- } \\
\text { tratamento }\end{array}$ & 15 & 3,400 \\
\hline$<12$ & $\begin{array}{l}\text { doença } \\
\text { medo } \\
\text { cuidado }\end{array}$ & $\begin{array}{c}10 \\
9 \\
9\end{array}$ & $\begin{array}{l}2,100 \\
2,889 \\
2,444\end{array}$ & $\begin{array}{l}\text { prevenção } \\
\text { morte }\end{array}$ & $\begin{array}{l}8 \\
6\end{array}$ & $\begin{array}{l}3,125 \\
3,500\end{array}$ \\
\hline
\end{tabular}

FIGURA 2: Análise do termo indutor "HIV/AIDS" evocado pelos profissionais de saúde que vivem com companheiro (a). Recife, PE, Brasil, 2013. 
prévios dos grupos sociais, tendo sido a AIDS, por muito tempo, associada a condutas desviantes ${ }^{17,18}$, gerando a omissão da sorologia a fim de evitar sofrer preconceitos. O fenômeno social e histórico da AIDS gerou diversas dúvidas, sentimentos e preconceitos que influenciaram o imaginário social e propiciaram concepções negativas acerca da doença estigmatizando-a e ocasionando o comprometimento da qualidade de vida das PVHA $^{19}$.

Nesse sentido, vale ressaltar que o estigma é definido como um rótulo, definição de estereótipos, separação, perda de status e discriminação em um contexto no qual o poder é exercido. Essa condição é bastante comum na sociedade atual e afeta um conjunto de grupos populacionais diversificados, pois os paradigmas sociais dominantes induzem ao aumento das desigualdades de saúde, tornando-se um problema para a população, pois atrapalham ou inibem o acesso a vários recursos estruturais, interpessoais e psicológicos que poderiam ser utilizados para evitar ou minimizar problemas de saúde, além de dificultar a eficácia das intervenções de saúde pública ${ }^{20}$.

É oportuno destacar que as atuais demandas de saúde requerem mudanças de paradigmas tanto nos serviços quanto na formação acadêmica dos profissionais de saúde ${ }^{21}$. Desse modo, essas constatações exigem um (re)pensar da formação e prática dos profissionais envolvidos, que ultrapasse a dicotomia entre informação e formação, o isolamento de saberes, a cristalização de práticas - e que seja capaz de fazer compreender a complexidade dos indivíduos e de suas relações cotidianas ${ }^{22}$.

No que concerne à expressão central tratamento, há interação positiva com os elementos periféricos adesão-tratamento e prevenção, traduzindo o saber técnico e científico dos profissionais acerca do HIV/ AIDS. As expressões ligadas ao tratamento remetem, sobretudo, à terapia medicamentosa e clínica, sugerindo o advento da terapia antirretroviral (TARV) como determinante para o prolongamento de vida das PVHA, assim como o aumento da expectativa de vida e melhoria da qualidade de vida.

Nesse contexto, salienta-se a cronicidade da AIDS por constituir uma realidade presente nos países que facilitam o acesso à TARV ${ }^{23}$. Partindo dessa alusão, a AIDS evoluiu para uma doença crônica, na era moderna, tornando os pacientes mais tolerantes à terapia. Além disso, o tratamento direciona-se para as comorbidades não relacionadas ao HIV, redução de riscos e cuidados preventivos ${ }^{24}$.

Em relação ao uso de antirretrovirais e o convívio com a doença, apontamentos sobre a cronicidade do HIV/AIDS configuram-se como novos desafios para as equipes de saúde, que devem estimular o tratamento contínuo e a aceitação da condição de soropositividade mediante uma sociedade que ainda percebe a doença como um problema do outro ${ }^{25}$.
Para tanto, no que diz respeito aos aspectos relacionais entre os quadrantes, o elemento periférico prevenção e de contraste cuidado apresentam conotação positiva e uma perspectiva dimensional de conhecimento, cuja associação ocorre pela possibilidade do convívio com a doença e adoção de condutas capazes de evitar a contaminação com o vírus. Sendo assim, está relacionada tanto à segurança do profissional ao lidar com o indivíduo soropositivo quanto ao seu relacionamento pessoal e social, além da realização de uma prática sexual segura pelos profissionais e pelos indivíduos em geral.

Estudo aponta a prevenção em um enfoque prático e real, cujas práticas preventivas vão além do uso do preservativo. Ressalta que as medidas de prevenção são fatores essenciais para o controle da epidemia, cabendo aos gestores e aos profissionais de saúde a transmissão de informações à população acerca dessas práticas ${ }^{26}$.

Corroborando essa premissa, a necessidade de prevenção por trabalhadores de saúde, além de convergir para a educação em saúde, pode estar associada à preocupação relacionada à transmissão viral ou reinfecção, capazes de induzir ao descompasso terapêutico ${ }^{17}$.

Quanto aos sentidos negativos acerca do objeto, pôde-se identificar, no terceiro e quarto quadrantes, os termos morte e medo, respectivamente, sendo a morte caracterizada pela dimensão de imagem, elaborada socialmente desde a identificação inicial da doença. Nesse contexto, cabe mencionar que o medo parece sugerir o receio do grupo investigado à exposição ao vírus e à possibilidade de infecção. Ainda, outra pesquisa salienta que o medo da doença associa-se ao preconceito, suscitando conflitos no relacionamento interpessoa ${ }^{11}$.

Os resultados deste estudo também reforçam aqueles encontrados em outra investigação, que demonstra o deslocamento do termo morte e o aparecimento do léxico doença crônica na zona de contraste da representação da AIDS, apontando uma mudança representacional observada nos últimos anos ${ }^{27}$.

Em contrapartida, o medo está intimamente ligado ao receio social de se contaminar com o vírus, devido a todas as particularidades evidenciadas no processo saúde-doença, além do medo da morte vivenciado por parte das PVHA.

As representações são criadas por pessoas e grupos a partir do processo de comunicação e cooperação e, ao adquirir vida própria, manifestam-se e formam novas representações. Esse processo de compartilhamento reforçado pela tradição constitui a realidade social e, assim, é possível modificar as atitudes de acordo com as representações fabricadas ou herdadas da sociedade ${ }^{28}$.

Acredita-se que o objeto representado implica algumas definições a partir de uma visão consensual da realidade compartilhada por um mesmo grupo e, embora seja divergente das significações construídas por outro 
grupo, pode servir para um processo de trocas cotidianas, que constituem a dinâmica das representações sociais ${ }^{29}$.

Nessa perspectiva, a evolução epidemiológica do HIV/AIDS e o acesso às informações denotam diversos conceitos, significados, sentimentos e ideias que vêm dando uma nova configuração à representação social da AIDS $^{26}$. Convém mencionar que a existência das representações ocorre pela necessidade da troca de informações entre as pessoas, tornando possível a compreensão dos problemas cotidianos apresentados, cujo enfrentamento requer ajustes de comportamento e domínio físico e cultural ${ }^{28}$.

No que diz respeito ao HIV/AIDS e situação de conjugalidade, a identidade conjugal envolve o compartilhamento de valores, posturas e cuidados, assim como está associada aos fatores externos ligados aos indivíduos ${ }^{30}$. O relacionamento fixo faz com que seus envolvidos acreditem que ele sustenta alguma forma de prevenção, pois a estabilidade na relação seria capaz de conferir uma proteção contra a infecção pelo vírus. Essas ideias permeiam o contexto da vulnerabilidade para o(a) companheiro(a), devido à confiança na fidelidade depositada na parceria sexual, o que nem sempre é observado na realidade ${ }^{11}$.

Desse modo, com relação à representação social do HIV/AIDS pelos profissionais de saúde que vivem com companheiro(a), foram evidenciadas, no quadro de quatro casas, características positivas relacionadas aos termos tratamento, adesão-tratamento, prevenção, cuidado, bem como características negativas determinadas pelos elementos preconceito, morte, medo. No entanto, essa representação não se diferenciou daquela observada no grupo geral (com e sem companheiro/a), o que faz supor a não variabilidade da construção do pensamento social em função dessa condição.

\section{ConClusão}

As representações sociais do HIV/AIDS pelos integrantes da equipe multiprofissional de saúde deste estudo sinalizaram aspectos importantes acerca do fenômeno, sendo possível identificar os seus significados expressos pelo conjunto dos profissionais e aqueles que vivem com companheiro(a).

Nesta investigação, os dados demonstraram que as representações sociais do HIV/AIDS, por parte dos profissionais com companheiro(a), foram condizentes às representações do grupo geral, no que diz respeito à relevância do aspecto dimensional de conhecimento sobre o objeto, constatando o enfoque clínico e biomédico acerca do fenômeno salientado pela abordagem clínica e medicamentosa, acompanhado da estigmatização da doença e dos seus afetados.

Percebe-se que os conteúdos imagético, atitudinal e de conhecimento acerca de um determinado objeto podem influenciar nos processos de constituição do pensamento social. Justificativas e argumentos que alimentam essa ideia são capazes de produzir sentidos e significados construídos socialmente em relação ao HIV/AIDS.

Os dados também propuseram conteúdos representacionais focalizados na concepção técnico- científica do objeto de estudo, associada à prática assistencial. Enfatiza-se a necessidade de desenvolver estudos com profissionais de saúde, pois, esse grupo, em especial os membros da equipe de enfermagem, constitui uma população vulnerável a danos e agravos à saúde, tendo em vista a carga horária exaustiva, as condições laborais e de estresse capazes de acarretar práticas assistenciais inadequadas, além de poderem interferir de modo insatisfatório nas relações conjugais dessas pessoas. Também é oportuno ressaltar a necessidade de ampliação de estudos que aprofundem as questões sobre conjugalidade nos diversos componentes sociais, a partir de diferentes proposições teóricas.

Ademais, o estudo apresentou algumas limitações e fragilidades relacionadas ao quantitativo de participantes, o que sugere a ampliação de pesquisas sobre representações sociais orientadas pela abordagem estrutural envolvendo um número mais expressivo de profissionais de saúde, em todos os níveis de atenção à saúde.

\section{REFERÊNCIAS}

1.Smith JH, Whiteside A. The history of aids exceptionalism. J Int AIDS Soc. 2010; 13:1-8.

2.Unites Nations Programme on HIV/AIDS (UNAIDS). World Health Organization. Global report: UNAIDS report on the global AIDS epidemic 2013. [cited in 2014 Jun 20]. Available from: http://www.unaids.org/en/media/unaids/contentassets/documents/epidemiology/2013/ gr2013/UNAIDS_Global_Report_2013_en.pdf.

3.Ministério da Saúde $(\mathrm{Br})$. Secretaria de Vigilância em Saúde. Departamento de DST, Aids e Hepatites Virais. Boletim Epidemiológico Aids e DST. 2013; 2(1).

4.Formozo GA, Oliveira DC. Representações sociais do cuidado prestado aos pacientes soropositivos ao HIV. Rev Bras Enferm. 2010; 63:230-7.

5.Féres-Carneiro T, Diniz Neto O. Construção e dissolução da conjugalidade: padrões relacionais. Paidéia. 2010; 20:269-78.

6.Scorsolini-Comin F, Santos MA. Relacionamentos afetivos na literatura científica: uma revisão integrativa sobre a noção de conjugalidade. Psicol Am Lat. 2010; 19:1-21.

7.Santos ÉI, Gomes AMT, Oliveira DC, Marques SC, Bernardes MMR, Felipe ICV. Representações sociais elaboradas por enfermeiros que cuidam de pessoas com HIV acerca de sua vulnerabilidade. Rev enferm UERJ. 2014; 22:303-8.

8.Oliveira DC, Gomes AMT, Marques SC, Penna LHG, Pontes APM, Sá CP et al. As transformações do cuidado de saúde e enfermagem em tempos de aids: representa- 
ções sociais e memórias de enfermeiros e profissionais de saúde no Brasil. Rio de Janeiro: Universidade do Estado do Rio de Janeiro; 2010.

9.Azevedo DM, Miranda FAN. Teoria das representações sociais e alceste: contribuições teórico-metodológicas na pesquisa qualitativa. Sau \& Transf Soc. 2012; 3:4-10.

10.Martinez EA, Souza SR, Tocantins FR. As contribuições das representações sociais para a investigação em saúde e enfermagem. Invest Educ Enferm. 2012; 30:101-7.

11.Gomes AMT, Oliveira DC, Santos ÉI, Santo CCE, Valois BRG, Pontes APM. As facetas do convívio com o hiv: formas de relações sociais e representações sociais da aids para pessoas soropositivas hospitalizadas. Esc Anna Nery. 2012; 16:111-20.

12.Oliveira DC, Marques SC, Gomes AMT, XZTeixeira MCTV. Análise das evocações livres: uma técnica de análise estrutural das representações sociais. In: Paredes AS. Perspectivas teórico-metodológicas em representações sociais. João Pessoa: Editora Universitária UFPB; 2005. p.573-603.

13. Wachelke J, Wolter R. Critérios de construção e relato da análise prototípica para representações sociais. Psicol Teor Pesqui. 2011; 27:521-6.

14.Gomes AMT, Oliveira DC, Sá CP. O sistema único de saúde na representação social de usuários: uma análise de sua estrutura. Rev Bras Enferm. 2011; 64:631-8.

15.Ministério da Saúde (Br). Conselho Nacional de Saúde. Resolução n ${ }^{\circ}$ 466/12 de 12 de dezembro de 2012. Brasília (DF): CNS; 2012.

16.Abric JC. Pratiques sociales et représentactions. Paris (Fr) Presses Universitaires de France; 1994.

17.Costa TL, Oliveira DC, Formozo GA. Representações sociais sobre pessoas com hiv/aids entre enfermeiros. Estud pesqui psicol. 2012; 12:242-59.

18.Gomes AMT, Silva ÉMP, Oliveira DC. Representações sociais da aids para as pessoas que vivem com hiv e suas interfaces cotidianas. Rev Latino-Am Enfermagem. 2011; 19:485-92.
19.Carvalho SM, Paes GO. A influência da estigmatização social em pessoas vivendo com hiv/aids. Cad saúde colet. 2011; 19:157-63.

20.Hatzenbuehler ML, Phelan JC, Link BG. Stigma as a fundamental cause of population health inequalities. Am J Public Health. 2013; 103:813-21.

21.Lima ICV, Galvão MTG, Paiva SS, Brito DMS. Ações de promoção da saúde em serviço de assistência especializada em HIV/Aids. Ciênc cuid saúde. 2011; 10:556-63. 22.Tura LRF, Madeira MC, Silva AO, Gaze R, Carvalho DM. Representações sociais de hepatites e profissionais de saúde: contribuições para um (re) pensar da formação. Ciênc cuid saúde. 2008; 7:207-15.

23.Polejack L, Seidl EMF. Monitoramento e avaliação da adesão ao tratamento antirretroviral para HIV/aids: desafios e possibilidades. Ciênc saúde coletiva. 2010; 15:1201-8.

24.Greene M, Justice AC, Lampiris HW, Valcour V. Management of human immunodeficiency virus infection in advanced age. JAMA. 2013; 309:1397-405.

25.Lago ELM, Maksud I, Gonçalves RS. A sorodiscordância para profissionais de saúde: estudo qualitativo da assistência em ambulatório de hiv/aids em município do estado do Rio de Janeiro. Temas psicol. 2013; 21:973-88.

26.Marques SC, Tyrrell MAR, Oliveira DC. Imagens e significados da aids entre usuárias dos serviços da rede básica de saúde do município do Rio de Janeiro. Psicol teor prát. 2009; 11:97-113.

27. Oliveira DC. Construção e transformação das representações sociais da aids e implicações para os cuidados de saúde. Rev Latino-Am Enfermagem. 2013; 21:276-86. 28.Moscovici S. Representações sociais: investigações em psicologia social. $8^{\mathrm{a}}$ ed. Petrópolis (RJ): Vozes; 2011. 29.Jodelet D. As representações sociais. Rio de Janeiro: EDUERJ; 2001.

30.Scorsolini-Comin F, Santos MA. Laços de família: a conjugalidade como dimensão de análise do desenvolvimento. Polêmica. 2011; 10:288-98. 